\title{
Examination along with Precise Mapping of Radio Frequency Pollution over Environment of Elementary School in Banja Luka
}

\author{
Zoran Popović1 Predrag Ilić $^{1 *}$, Suzana Gotovac Atlagić ${ }^{2}$, Slađana Rikić1, \\ Branko Radović ${ }^{1}$ \\ ${ }^{1}$ PSRI Institute for Protection and Ecology of the Republic of Srpska, Vidovdanska 43, Banja Luka, \\ Republic of Srpska, Bosnia and Herzegovina \\ ${ }^{2}$ Faculty of Natural Sciences and Mathematics, University of Banja Luka, Dr. Mladen Stojanović 2, \\ Banja Luka, Bosnia and Herzegovina
}

Received: 14 February 2021

Accepted: 29 March 2021

\begin{abstract}
Rapid telecommunications developing during previous decades is followed by novel technology systems as well as enlargement of environmental non-ionized radiation level. Emitted electromagnetic signals from base stations are shifted to the higher frequencies range with limited penetrability, requiring additional strength which implicates increasing risk for human health. The present research shows the stage of electromagnetic pollution from exposed locations in the vicinity of the elementary school in Banja Luka, obtained both by experimental measurements and theoretical estimations. For theoretical prediction, a new upgraded software was developed for visualization in two and three dimensions of real space, where calculations are performed including all fundamental characteristics of the antenna along with environment characteristics.
\end{abstract}

Keywords: high frequency non-ionized radiation, exposure, environmental protection

\section{Introduction}

The world is currently experiencing drastic development of telecommunication technologies, where basic carriers are electromagnetic waves [1]. In the span of only a few years, several technologies have been raised up into commercial use, having enormous economic as well as social impact all over the world.

*e-mail: predrag.ilic@institutzei.net
All developed mobile telecommunications technologies used until now will be also soon installed over the Bosnia and Herzegovina. Among them are technologies GSM 900, GSM 1800, UMTS 900, UMTS 2100 and recently the new set up four generation technology LTE 800 and 1800. The present article therefore focuses on analysis and examination of exposure to the high frequency non-ionized radiation of the school area in Banja Luka city by different methodologies. Banja Luka is Bosnian second largest city, capital of the Republic of Srpska entity, where over $10 \%$ of the country's population is situated. The subject 
of the study was chosen since people are more and permanently exposed to electromagnetic pollution and this region is no exception. Along with the increasing chemical, biological and other pollutants found in water or food, as well as physical phenomena like ultraviolet radiation, electromagnetic pollution is one of certainly large health risk. People become more and permanently exposed to electromagnetic pollution. Today, sources of these harmful effects are widespread due to the telecommunications development. In accordance with the high market demands, high frequency electromagnetic field guided telecommunication signals becoming increasingly dense. Rapid advances in telecommunication technologies are followed by the earlier exposition of children to this kind of pollution. Exposure and sensitivity of children to the wide range of electromagnetic spectra is investigated as a possible cause of increasing in grave childhood illnesses like leukemia. Frequently appearing of difficult illness like childhood leukemia is still investigated as a possibility that children are sensitive to some or to the wide range of electromagnetic spectrum [2, 3]. The World Health Organization (WHO) emphasizes the importance of research on radio frequency influence on health and development of the appropriate recommendation [4]. Based on consistent epidemiological data association between childhood leukemia and radiation is classified by the International Agency for Research on Cancer (IARC).

New generation emitters of $4 \mathrm{G}$ technology, are more frequently used in the area of Western Balkans, incorporated within already existing based stations. As a consequence, newly installed technology is likely to have additional impact on contamination through living and working environmental, however, the reports in the literature on this problem are still scarce.

It is reported that RF electromagnetic field radiation exposure can change neurotransmitter functions, blood-brain barrier, morphology, electrophysiology, cellular metabolism, calcium efflux, and gene and protein expression in certain types of cells even at low intensities [5], since the biological consequences of such changes remain unclear yet. High frequency electromagnetic field influence on living organisms, especially effects on human tissues are still unknown or not yet completely light upon. Radio frequency (RF) fields have more hazards impact on children because their nervous system is generally with grater susceptibility. Since children's brains are more conductive than those of adults, electric field penetrates deeper relative to the size of their head [4].

The quantities of carried frequency of the novel technologies are constantly increasing, interacting with different matter. Therefore, when one talks about environmental hazards, here related with human tissue, mainly composed of water, it cannot be explained commonly in accordance with classical physics. Effects following the interaction with RF electromagnetic waves become more pronounced and require different scientific approach. For example, recent study presented in [6] gives overview of influence of high frequency mobile signal on neurology status of permanently exposed mice. Pronounced biological consequences are confirmed. It was found that exposure to RF field led to damage of neurons since mice displayed hyperactivitylike behaviour.

Upcoming novel generation of telecommunication technology known as 5G, with frequency range which goes over $24 \mathrm{GHz}$, is covering frequency of boiling water and is almost completely absorbed during interaction with matter. Therefore, the present research should give the suggestion on methodology in research in the influence of these technologies on overall pollution and point out to the potential hazards to human health.

\section{Experimental}

Two different approaches for determination of level of high frequencies non-ionized radiation were applied at every investigated location. First approach is modelling of electromagnetic field intensity in vicinity of radiation sources. Predicting places with potentially high level of field intensity in some part of the space around emitters, is not an easy task, even in the far field regions. Theoretical predictions are found to be necessary in order to determine the points with the potentially highest intensities of electromagnetic environmental pollution over examine location and evaluate their levels. The application of theoretical predictions as a preparation for experimental testing of environmental electromagnetic pollution near already installed base station is not the only benefit achieved by this method. This way, levels of RF intensities over the wide area surrounding base stations could be predicted, even long before the telecommunication equipment or some other antennas are installed at the particular location. Most of the risks related to environmental pollution by installation of planned base station should be avoidable through theoretical prediction presented in this study. This is of particular importance for the locations where installation of base stations are planned in the future, but which already contain sources of high frequency non-ionizing radiation. There will be even more increase in requirements for this kind of predictions and permanent control of actual state by measuring since development of the telecommunication systems is continuous. Large factor is also increase in user requests, like increasing of the speed of internet data or flow of mobile connections.

Second, more classical approach in investigation of environmental pollution is a direct detection via measurement with appropriate calibrated instruments. In present research, the state-of-the-art sophisticated equipment was used, with all key features for testing broad range of widespread generated electromagnetic signals. Used devices, possessing antenna and the 
analyser together with appropriate software, are able to detect and analyze signals of all existing modern telecommunications technologies as well as all emitted television and radio broadcast signals.

\section{Theoretical Predictions and Software Solution}

For typical calculations of environmental RF exposure fundamental properties of antenna are the essential data. They are mostly given by telecommunication operators and constructors, and published as technical specifications. In order to predict state with maximal exposure, the largest output emitted power is necessary as input parameter for calculation. Also, specification must contain following parameters: antenna gain, allocated carriers and losses in conductors. Mostly, used antennas in telecommunication technology have anisotropic characteristic, meaning that radiation power density of RF depends on the direction of spreading. Horizontal/vertical radiation patterns, here labeled with $\mathrm{g}_{H(V)}(\Theta, \varphi)$, are given in spherical coordinates, where $\Theta$ is elevation while $\varphi$ is azimuth angle. Radiation patterns contain side and minor lobes, with relatively weak portion of radiation emitted in undesired directions. Total pattern is used to evaluate antenna emission anisotropic over the entire space and deviation from spherical isotropic emission, mathematically given as:

$$
\mathrm{g}(\Theta, \varphi)=g_{H}(\Theta)+g_{V}(\varphi)
$$

According to the shape of each individual pattern (horizontal and vertical) surface with equal radiation flux are expected to be with many lobes.

Except the mentioned fundamental properties of antenna, additional relevant data for estimation of radiation level are the geometrical characteristics: height, orientation and mechanical tilt angle. All of these parameters are incorporated in calculations algorithm, implemented through designed software

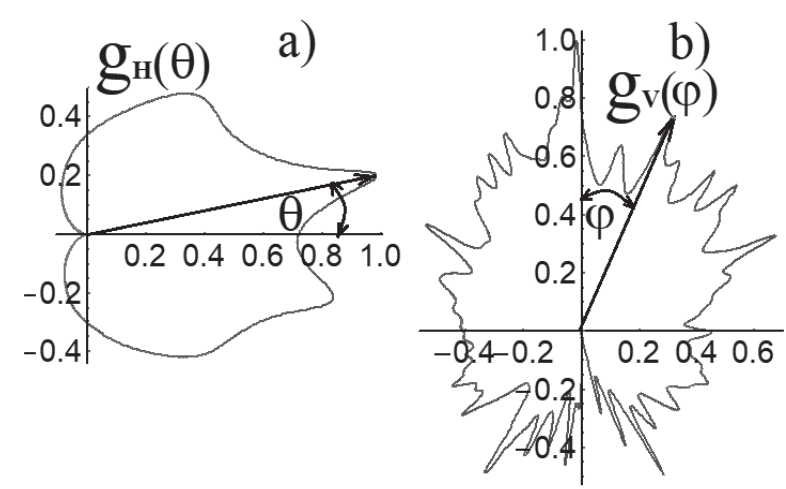

Fig. 1. Horizontal a) and vertical pattern b) of antenna A264518S0_1748_X_CO_P45, installed on the roof at technical inspection near elementary school Jovan Cvijić in Banja Luka. for calculation levels of high frequency non-ionized radiation around installed antennas as well. Besides, the software is engineered to map partial contributions levels and their RF field strength from individual emitter or cumulatively as a sum of all individual emitter set up at the given location. Advantage of the presented visualization is a possibility of two (2D) and three (3D) dimensional environmental mapping of high frequency electromagnetic pollution. The evaluations of the exposure levels to electromagnetic fields generated by GSM 900, GSM 1800 and 3G base stations in urban areas, including the determination of the minimum safe distances for population and occupational exposure, are computationally obtained and analysed in earlier studies, e.g. Hamiti et al. (2016) [7].

One of the relevant parameters, given from telecommunication operator is effective isotropic radiated power labeled as EIRP, usually given in $\mathrm{dBm}$ units. This quantity is obtained from relation

$$
E I R P=P_{T}+G_{A}-L_{C}
$$

...where $P_{T}$ is of transmtter given in $\mathrm{dBm}, G_{A}$ is antenna gain in $\mathrm{dBi}$ and losses in cable output power denoted with $L_{C}$ in $\mathrm{dB}$ [8-10].

The intensity of emitted RF radiation or power density from $i$-th source in the certain point of space with spherical coordinates $(r, \Theta, \varphi)$ is given as

$$
S i(r, \theta, \varphi)=d P / r^{2} d \Omega
$$

...where $r$ is radii from source to observed point, $\mathrm{d} \Omega$ is infinitesimal solid angle through which radiation of power $d P$ passes, and is calculated as in $[7,8]$. Power of RF radiation, passing through elementary surface is part of anisotropic redistribution in accordance with antenna pattern.

Signals from different sources are considered as uncorrelated, discussed in [8], such that total power density at the selected point is given as

$$
S_{\text {total }}(r, \theta, \varphi)=\sum_{i} S_{i}(r, \theta, \varphi)
$$

Different quantities, relevant for visualization of exposure level like intensity of total electric field, partial contribution from different set up technology, total exposure, and average dose e.g. can be represented as projection on real satellite map, previously shown in [11]. This method represents improved technical and computational solution compared with previous, presented in that literature. Designed software now is used as a tool for systematic examination of environmental exposure to the RF electromagnetic field. As it is demonstrated in Fig. 2, this analysis offers overview of electromagnetic pollution state around specified area. Exposure below limited for general population at $2 \mathrm{~m}$ above ground, between 0.008 and 0.32 divided in 4 levels is shown on $2 \mathrm{D}$ diagram in Fig. 2. School, kindergarten as well as residence around 


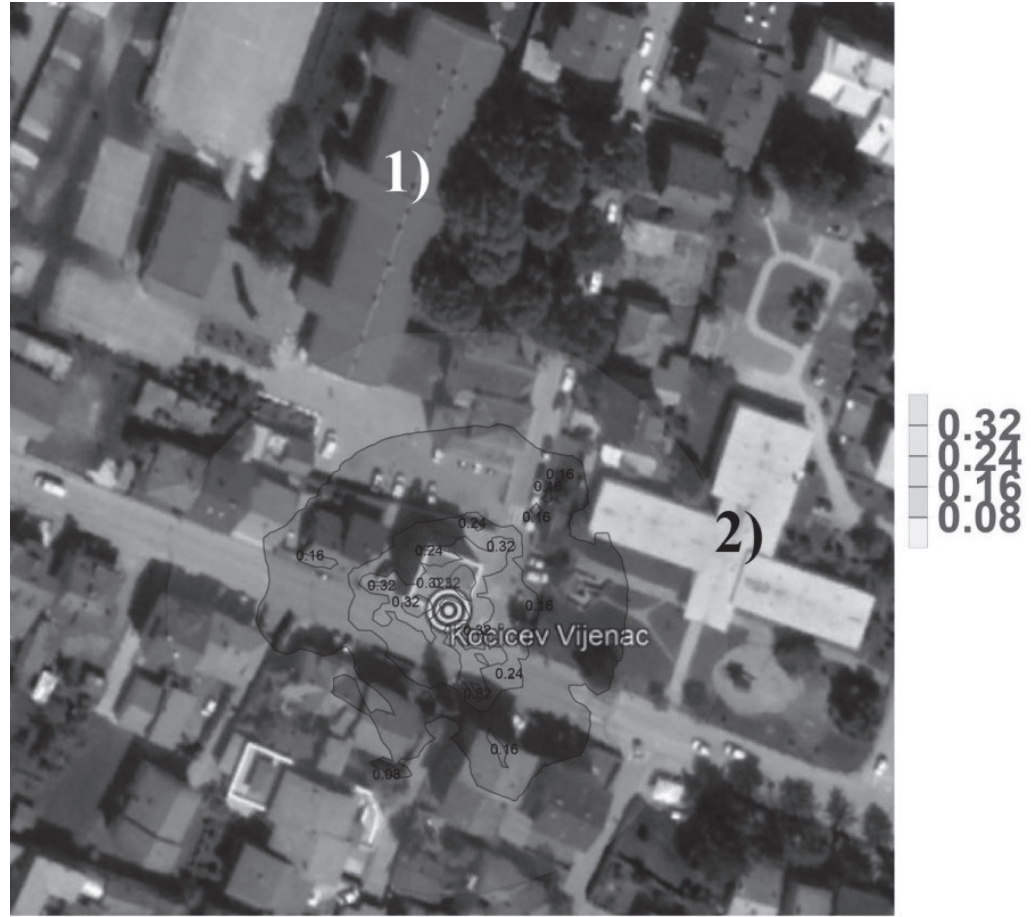

Fig. 2. Two dimensional visualization of environmental exposure in vicinity of elementary school Jovan Cvijić in Banja Luka, where elementary school and kindergarten are labelled with 1) and 2) respectively. Level 1 of exposure corresponds to the limit value for general population.

examined area are exposed in very small amount to RF electromagnetic source, almost insignificantly. However, from Fig. 2 it is not clear are there parts of target objects in the level of the horizontal plane with antenna which are exposed significantly. It would be important to consider if there is any lob that spread indirectly and reach up to these objects. Earlier, it have been discussed in the literature [11] that there is a need to develop the technology for a radio frequency mapping system which could be deployed over populated areas such as cities, industrial zones and other inhabited places. Detail analysis, presented here, offers three dimensional representation of exposure in vicinity of RF emitters. It shows ray tracing independently on anisotropy, position and orientation of antenna.

The advantage of describing the spread of electromagnetic pollution over environment through three dimensional displays, is reflected by the possibility to obtain complete comprehension and reach any intensity of RF field, and to obtain detection of particular places in free space where exposition is expected.

The improved upgrade of software for visualization of environmental exposure to the RF radiation is now implemented and applied during studies of electromagnetic pollution in the work of the present institution. It offers detailed inspection of locations containing sources of high frequency electromagnetic fields like shown in Fig. 3. Main preference of this visualization compared with previous, planar display of exposure, is that present new method gives a possibility to observe from different point, angles of view and arbitrarily enlarged parts of the space exposed with certain intensity of radiation. Firstly, on these diagrams associated objects should be identified and a satellite map with blocks on 3D illustration constructed. 3D maps focus on the relevant places of interest such as: school, school playground, kindergarten or residential houses, namely, mainly places where there is a possibility that general population, primarily children, could be exposed to $\mathrm{RF}$ radiation. When those objects are identified, their positions from different angles, point of view and aspect ratio, and are presented in relation with area containing certain degree of radiation. Implemented code for this theoretical calculation and estimation of RF field strength allows separation and denote the space around the emitter, one or more on actual location, with an arbitrary level of intensity. In Fig. 3 and 4 labels areas around antenna, set up in vicinity of primarily school and kindergarten, where potential exposure of children is examined. Exposed space around emitters is represented as a transparent cloud with different colors, from blue/green corresponding to lower, up to red colour for upper limit of given RF intensity, positioned closer to source of radiation.

\section{Results of Measurement Near Elementary School Jovan Cvijić}

There were several steps during the procedure of processing results of measurement. All of them were in agreement with legislation and basic standard 

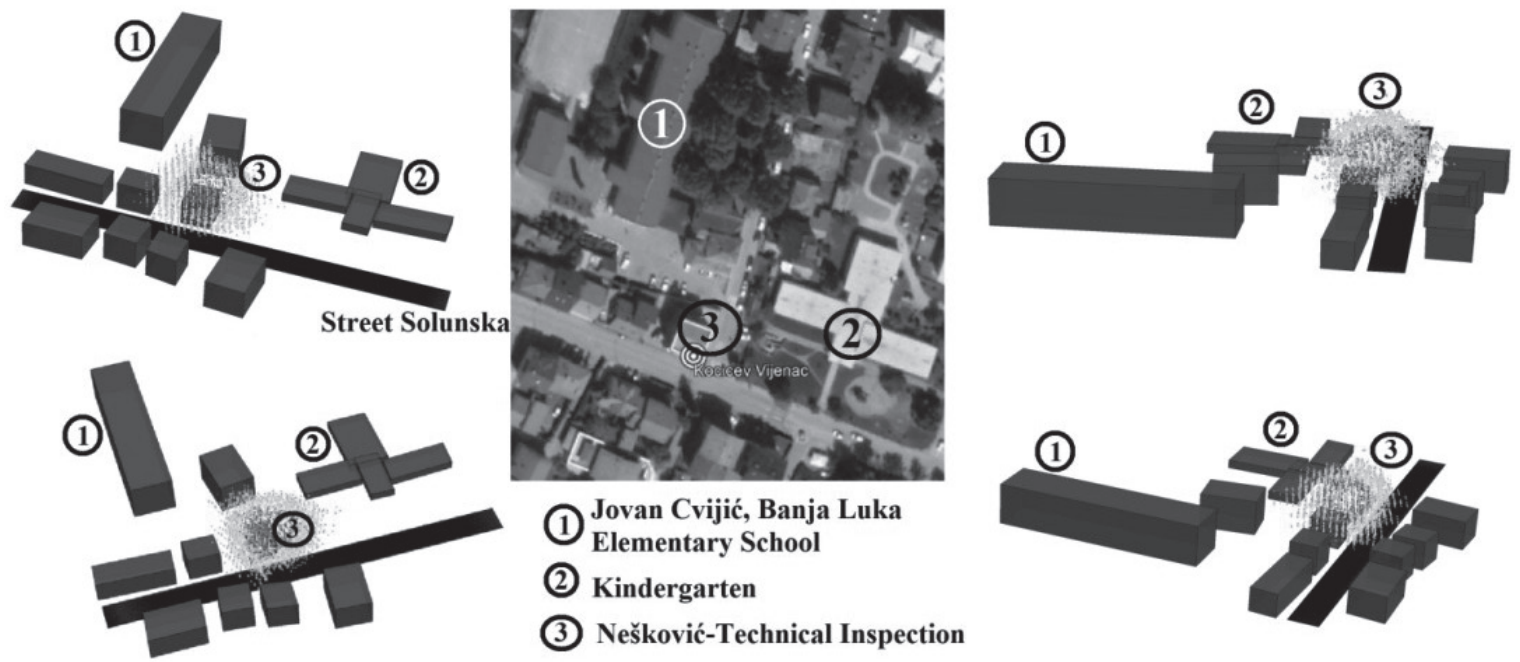

Fig. 3. Painted cloud around base station, positioned at roof of the red block, reaches out environmental places with electromagnetic pollution up to $30 \%$ of limit value for general population. Relevant objects with appropriate assignation on satellite map (1- Elementary school Jovan Cvijić Banja Luka, 2- kindergarten, 3- building of technical inspection carrier of base station), exposed to high frequency radiation from installed base station, are shown on images of three dimensional objects layout from different point of view.

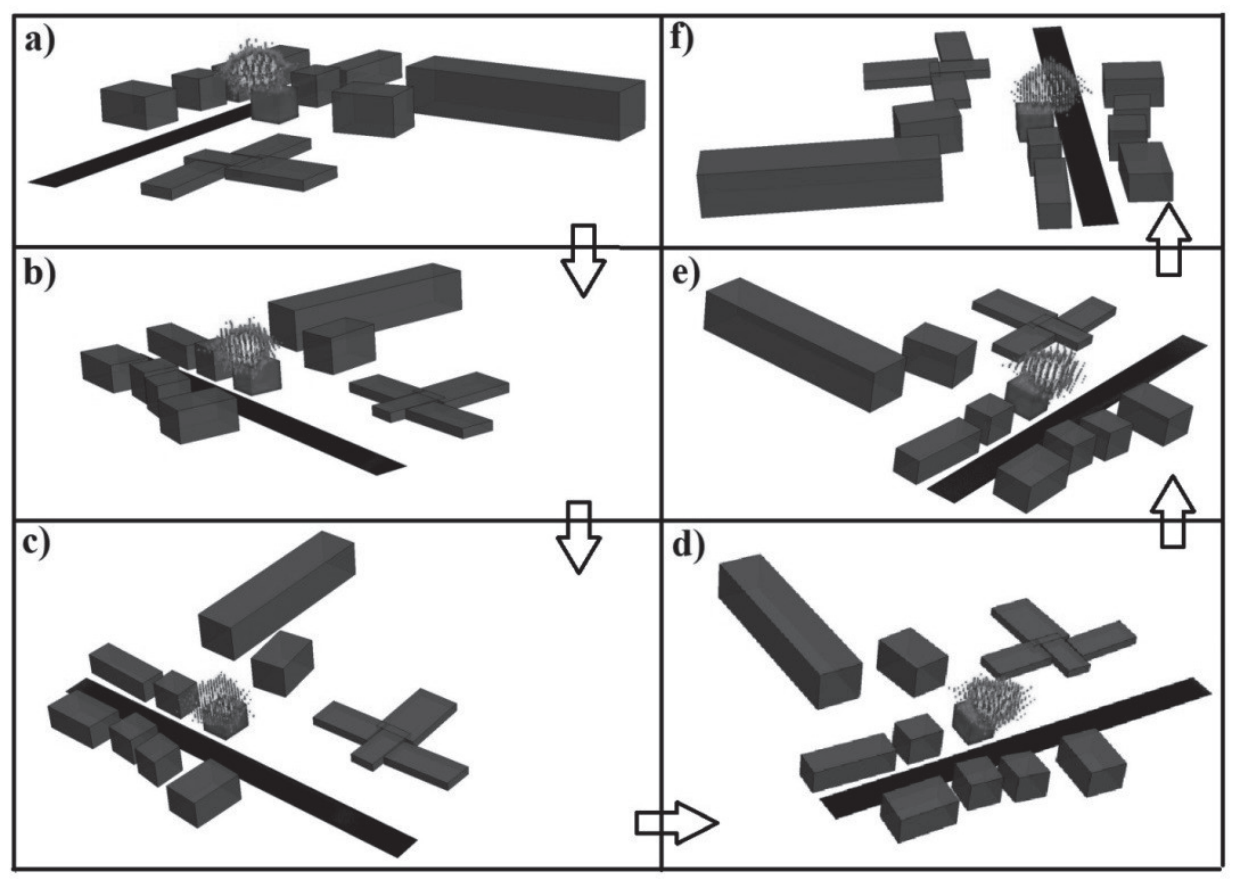

Fig. 4. Space around building with base station on the roof (block painted with red), exposed over limits proposed for general public is shown with painted cloud in particular part of three dimensional real space. Spatial range of exposure limits to non-ionized radiation emitted from base station (installed at red block) at location as a size of the painted cloud, inspected from six different points of view from images a) to f) are shown.

for measurement and procedure of calculation BAS EN 50413:2010 and BAS EN 50413/A1:2014, and were performed in accordance with the laboratory accreditation. After theoretical preparation, computationally selected measurements points where higher levels of RF radiation emitted from local base station were expected measurements have been done using the calibrated equipment.
Equipment used in measurements consisted of isotropic antenna, type EMF SYSTEM TRAGBAR, manufactured by ROHDE\& SCHWARZ (R\&S). It is sensitive on electric field between $1 \mathrm{mV} / \mathrm{m}$ and $100 \mathrm{~V} / \mathrm{m}$ in the frequency range from $30 \mathrm{MHz}$ up to $3 \mathrm{GHz}$. In addition, indispensable part of measurement equipment is handheld spectrum analyzer produced by R\&S, which allows working in frequency range between 


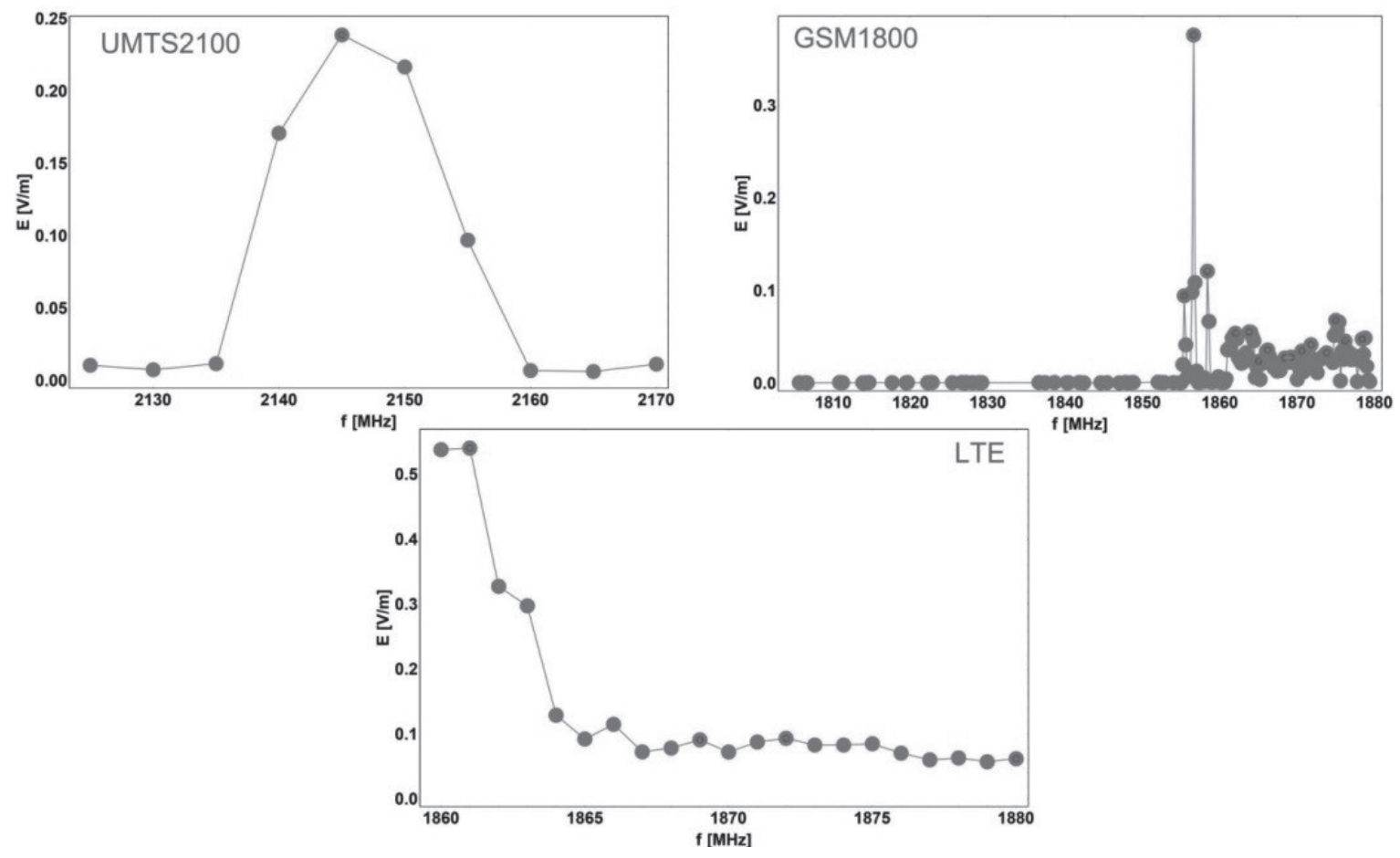

Fig. 5. Spectra of respective technologies: UMTS 2100 (upper left), GSM1800 (upper right) and LTE 1800 (down), measured in the maximum exposed point $2 \mathrm{~m}$ above ground in vicinity of antenna.

$100 \mathrm{kHz}$ and $8 \mathrm{GHz}$, characterized by high sensitivity $(<-141 \mathrm{dBm}, 1 \mathrm{~Hz})$ and low measurements uncertainty $(<1 \mathrm{~dB})$. Used analyser was FSH8 type, which offers many benefits like channel power measurements, analysis of transmit signals, vector network analysis, highly accurate power measurements and all these with easy operation.

Measurements started with the fast inspection, applying the scanning of total electric field strength generated from sources in vicinity, as demanded by Regulations. Detection of sources at particular locations was carried on with the analyser and the program package FSH View. With these tools it was possible to detect appropriate signals together with their frequencies and levels. After locating relevant existing signals, with significant field strength, their spectrum was recorded as a field intensity dependence on frequency. For detecting and analysing the spectrum of target signals, the RFEX software was used. Primarily, it was necessary to define the packages for all telecommunication technologies or other broadcast television of radio signal. The report should contain relevant information about measuring technology consisting of duration time of measuring cycles, frequency step, threshold etc.

All obtained results of RF fields are firstly extended for uncertainty, necessary due to existing of unavoidable errors. Several significant and independent impacts are involved in estimation of total uncertainty quantification. Every procedure contained several types of independent, unavoidable and unremarkable sources of errors. Detected and classified relevant sources of errors are: uncertainty of amplitude indication on display of analyzer, linearity of response, noise level, uncertainty impact from antenna, field perturbation and personal errors. As a cumulative effect of all mentioned sources, expanding factor is obtained to the level of reliability of $95 \%$. After measurement, the obtained results firstly were extended up to the measurable uncertainty. In order to estimate the worst possible scenario, obtained field values were magnified by $31.73 \%$.

Afterwards, results extended for uncertainty needed to be properly corrected relative to the used technology. It means that they needed to be increased additionally in accordance with range of measurement. In particular, for GSM technology final values of electric field used to obtained exposure, were obtained by multiplying extended values by corresponding factor $\sqrt{ }_{n_{T R X}}$ where $n_{T R X}$ is the number of GSM channels downlinks.

Exposures for all individual technologies derived from measured values are calculated, as well as the cumulative ones, obtained as total exposure at given point. Results of measurement at examined location shown in Fig. 5 are in good agreement with theoretical predictions in the sense of matches of obtained exposure level. Consideration of safety over area around installed base station, low strength of individual technology fields as well as total exposure are found. In particular, after applying all necessary extensions related to the measurement uncertainty and corresponding factor requirements for all involved technologies, obtained values of exposition are in the range allowed for general population. 


\section{Conclusions}

The analysis of the risks, due to the electromagnetic environmental pollution, conducted in accordance with legislation and valid standards confirmed minor risk for general population at investigated area in vicinity of elementary school. Engineering of novel computational technique implemented as 3D visualization through upgraded software offers significant improvement in analysis of space distribution of this type of pollution. The presented technique made it possible to determine measurement points, to detect potential hot spots at examined location and also to predict the saturation of exposure in accordance with proposed levels. The main contribution to the electromagnetic pollution mostly originated from the base stations, becoming stronger with new technology installed. All emitters around schools, kindergartens or any other places where children are potentially widely exposed, even to a small amount of electromagnetic field radiation, should be properly constructed so that risk of exposure is minimized. This could be managed by selecting optimal parameters of antenna, including lowering emitted power, installation of antenna on appropriate height, pick up optimal orientation and mechanical tilt to avoid directly irradiation of target object.

\section{Acknowledgements}

This research is funded by the Ministry for Scientific and Technological Development, Higher Education and Information Society of Republic of Srpska (19/6020/961-95/18) in the frame of project "Investigation of high frequency non-ionized electromagnetic radiation exposure of the school in Banja Luka, located in the vicinity of the telecommunication base station". The present research was realized entirely with the equipment from the PSRI Institute for Protection and Ecology of the Republic of Srpska.

\section{Conflict of Interest}

The authors declare no conflict of interest.

\section{References}

1. RAMAKRISHNAN N., MALARKKAN S. Radio frequency smog reduction from future heterogeneous base station, Biomed. Res. An Int. J. Med. Sci., Special Issue. S38, 2016.

2. KHEIFETS L., REPACHOLI M., SAUNDERS R., VAN DEVENTER E. The sensitivity of children to electromagnetic fields, Pediatrics. 116 (2), e303, 2005.

3. HARDELL L., World Health Organization, radiofrequency radiation and health-a hard nut to crack, Int. J. Oncol. 51 (2), 405, 2017.

4. WIEDEMANN P., SCHÜTZ H. Children's health and RF EMF exposure. Views from a risk assessment and risk communication perspective, Wien. Med. Wochenschr. 161 (9-10), 226, 2011.

5. SIVANI S. SUDARSANAM D. Impacts of radiofrequency electromagnetic field (RF-EMF) from cell phone towers and wireless devices on biosystem and ecosystem-a review, Biol. Med. 4 (4), 202, 2012.

6. KIM J.H., YU D.H., HUH Y.H., LEE E.H., KIM H.G., KIM H.R. Long-term exposure to $835 \mathrm{MHz}$ RF-EMF induces hyperactivity, autophagy and demyelination in the cortical neurons of mice, Sci. Rep. 7 (1), 1, 2017.

7. HAMITI E., IBRANI M., AHMA L., SHALA V., HALILI R. Comparative analysis of electromagnetic field exposure levels and determination of the minimum safe distances from mobile-phone base stations in urban areas, Prog. Electromagn. Res. 50, 117, 2016.

8. POLJAK D. Human exposure to the radiation of base station antenna systems - incident field dosimetry, Sigurnost. 54 (2), 173, 2012.

9. OH S.S., LEE Y.H. An EIRP measurement method for base-station antennas using field strengths measured along a single straight line, Int. J. Antenn. Propag. 2013 (ID 742636), 1, 2013.

10. JANG B. J., MOON S. W., YOON H. Maximum Eirp and EMF Estimation Based on Over-the-Air Measurements of Wcdma Pilot Channel, Prog. Electromagn. Res., 31, 215, 2012.

11. POPOVIĆ Z., ILIĆ P., MIROŠLJEVIĆ R., GOTOVACATLAGIĆ S. Exposure to non-ionizing radiation of area in urban zone of the Banja Luka city, Arch. Tech. Sci. 20 (1), 81, 2019.

12. CHACZKO Z., ABOURA K., AGBINYA J. Software engineering for mapping radio frequency pollution, Int. J. Electron. Telecommun. 56 (2), 129, 2010. 